\title{
Energy Partition Oscillator and Necessary and Sufficient Conditions of Energy Localization
}

\author{
V.N. Pilipchuk ${ }^{1, a}$ \\ Wayne State University, Detroit, Michigan, U.S.A.
}

\begin{abstract}
A strongly nonlinear conservative oscillator describing the dynamics of energy partition between two identical linearly coupled Duffing oscillators is introduced and analyzed. Temporal shapes of such oscillator are close to harmonic when the initial energy disbalance between the interacting Duffing oscillators is relatively small. However the oscillator becomes strongly nonlinear as the amplitude of energy exchange increases. It is shown nevertheless that the oscillator is exactly solvable and, as a result, the entire first order averaging system, describing the dynamics of coupled Duffing oscillators, admits exact analytical solution. Based on the first integral of the energy partition oscillator, necessary and sufficient conditions of energy localization are obtained in terms of the initial states of original system.
\end{abstract}

\section{Introduction}

The present paper deals with the problem of energy exchange between nonlinear oscillators due to their resonance interaction through a relatively weak elastic coupling. The system is assumed to be conservative and autonomous. During normal mode motions [1], the energy partition between the oscillators from one cycle to another is fixed so that there in no energy flow between the oscillators. However, when the initial states of oscillators are out of compliance with any of the system normal modes, then the oscillators slowly exchange by some portion or even all of the energy in a beat-wise way. Such beating phenomena have been in focus of nonlinear physics and physical mechanics for few decades by very different theoretical and practical reasons; see, for instance references [2], [3]. Usually, the beat dynamics are described through the transition to amplitude-angle or similar coordinates and then averaging the new equations over one cycle of vibration. After such preliminary manipulations, the differential equations of motion may appear to have one or may be several integrals according to the level of system' symmetry [3]. These types of integrals actually provide the description for the energy exchange between the interacting oscillators. Recently, based on the detailed parametric study of phase trajectories the notion of limiting phase trajectory (LPT) was introduced by Manevitch [4], [5]. It was noticed that, in the limit when the entire energy of the system swings from one oscillator to another, the descriptive phase angles resemble state variables of impact oscillators with non-smooth temporal shapes. The importance of this observation is that it provides asymptotic simplifications for the cases exactly opposite to the normal modes. Further, analytical algorithms of nonsmooth temporal transformations [6], [7] where adapted to

\footnotetext{
${ }^{\mathrm{a}}$ e-mail: pilipchuk@wayne.edu
}

build approximate analytical LPT solutions [8], [9], [10]; see also references therein.

In the present work, it is shown that the phase variable, which determines the energy partition (EP) between the two Duffing oscillators, is always described by another conservative oscillator for any intensity of the energy exchange, regardless the LPT limit. Moreover, such oscillator is exactly solvable and admits special cases described explicitly in terms of elementary functions. Finally, it is shown that first integral of the EP oscillator completely determines necessary and sufficient conditions for the energy localization effect.

\section{Nonlinear beat equations}

Let us consider a system of two identical linearly coupled unit-mass oscillators described by the Hamiltonian

$$
H=\frac{1}{2}\left(v_{1}^{2}+v_{2}^{2}\right)+\Pi\left(u_{1}\right)+\Pi\left(u_{2}\right)+\frac{1}{2} b\left(u_{1}-u_{2}\right)^{2}
$$

where $u_{i}$ and $v_{i}(i=1,2)$ are the coordinates and linear momenta, respectively, $\Pi\left(u_{i}\right)$ is an even analytic function describing the potential energy for each of the two oscillators, and $b$ is the coupling stiffness, which is assumed to be relatively week, $b / \Pi^{\prime \prime}(0) \ll 1$.

Introducing the parameters, $\Omega=\sqrt{\Pi^{\prime \prime}(0)+b}$ and $\varepsilon=b \Omega^{-2}$, and subtracting the parabolic component from the potential energy,

$$
\varepsilon U(u)=\Pi(u)-\frac{1}{2} \Pi^{\prime \prime}(0) u^{2}
$$

brings Hamiltonian (1) to the form

$$
\begin{aligned}
H= & \frac{1}{2}\left(v_{1}^{2}+v_{2}^{2}\right)+\frac{1}{2} \Omega^{2}\left(u_{1}^{2}+u_{2}^{2}\right) \\
& +\varepsilon\left[U\left(u_{1}\right)-\Omega^{2} u_{1} u_{2}+U\left(u_{2}\right)\right]
\end{aligned}
$$


The form of Hamiltonian (3) incorporates the additional assumption that both nonlinearity and coupling are of the same order of magnitude, $\varepsilon$. Note also that the coupling is represented now in somewhat canonical form after the non-coupled terms of the interaction energy (1), $b u_{i}^{2} / 2$, have been associated with the corresponding oscillators. The differential equations of motion are given by

$$
\dot{u}_{i}=\frac{\partial H}{\partial v_{i}}, \quad \dot{v}_{i}=-\frac{\partial H}{\partial u_{i}}
$$

or

$$
\begin{aligned}
& \dot{u}_{1}=v_{1} \\
& \dot{u}_{2}=v_{2} \\
& \dot{v}_{1}=-\Omega^{2} u_{1}+\varepsilon\left[\Omega^{2} u_{2}-U^{\prime}\left(u_{1}\right)\right] \\
& \dot{v}_{2}=-\Omega^{2} u_{2}+\varepsilon\left[\Omega^{2} u_{1}-U^{\prime}\left(u_{2}\right)\right]
\end{aligned}
$$

As $\varepsilon \rightarrow 0$, system (5) degenerates into two identical harmonic oscillators whose total energies are separately conserved. At non-zero $\varepsilon$, the oscillators become non-linear and interact with each other in such a way that one of the oscillators is loaded proportionally to the displacement of another oscillator. Since system (5) is perfectly symmetric and conservative, it is reasonable to assume a relatively slow energy exchange between the oscillators. In order to describe the corresponding process in physically meaningful terms, let us introduce a new set of variables as follows

$$
\begin{gathered}
\left\{u_{1}, v_{1}, u_{2}, v_{2}\right\}->\{K(t), \theta(t), \delta(t), \Delta(t)\}: \\
u_{1}=\sqrt{K} \cos \left(\frac{\theta}{2}+\frac{\pi}{4}\right) \cos \delta \\
v_{1}=-\sqrt{K} \Omega \cos \left(\frac{\theta}{2}+\frac{\pi}{4}\right) \sin \delta \\
u_{2}=-\sqrt{K} \sin \left(\frac{\theta}{2}+\frac{\pi}{4}\right) \cos (\delta+\Delta) \\
v_{2}=\sqrt{K} \Omega \sin \left(\frac{\theta}{2}+\frac{\pi}{4}\right) \sin (\delta+\Delta)
\end{gathered}
$$

In case $K, \theta$, and $\Delta$ are constant, and $\delta=\Omega t$, expressions (6) represent the exact general solution of the decoupled set of harmonic oscillators (5), $\varepsilon=$ 0 . Therefore, relationships (6) implement the idea of parameter variations compensating the perturbation, when $\varepsilon \neq 0$. In order to track the oscillator energies during the vibration process, let us introduce quantities

$$
\begin{aligned}
& E_{1}=\frac{1}{2}\left(v_{1}^{2}+\Omega^{2} u_{1}^{2}\right)=\frac{1}{2} E_{0}(1-\sin \theta) \\
& E_{2}=\frac{1}{2}\left(v_{2}^{2}+\Omega^{2} u_{2}^{2}\right)=\frac{1}{2} E_{0}(1+\sin \theta)
\end{aligned}
$$

where

$$
E_{0}=E_{1}+E_{2}=\frac{1}{2} \Omega^{2} K
$$

Expressions (7) and (8) clarify physical meaning of the variables $K$ and $\theta$ participating in transformation (6), where the other two variables, $\delta$ and $\Delta$, determine phases of the vibrating oscillators. In particular,
$K$ is proportional to the total energy of the decoupled and linearized oscillators, whereas the phase $\theta$ characterizes the energy partition between the oscillators. If $\varepsilon \neq 0$, then the energy parameter $K$ shows small temporal fluctuations due to coupling and nonlinear terms in (5). Nevertheless expressions (7) and (8) can still be used as energy related quantities for characterization of the energy exchange between the oscillators. As follows from (7), the interval $-\pi / 2 \leq \theta \leq \pi / 2$, on which $E_{0} \geq E_{1} \geq 0$ and $0 \leq E_{2} \leq E_{0}$, is sufficient to fully characterize the energy partition between the oscillators. In particular, the case $\theta=0$ corresponds to equipartition, $E_{1}=E_{2}$, which takes place when the particles oscillate either out-of-phase $(\Delta=0)$ or in-phase $(\Delta=\pi)$ according to the sign convention in (6). Although next few steps can be passed with a general power series representation for the potential energy function, $U\left(u_{i}\right)$, let us assume the monomial form, $U\left(u_{i}\right)=\alpha u_{i}^{4} / 4$.

In order to conduct transition to the new variables, let us substitute (6) in (5), then solve the set of equations with respect to the derivatives, and apply the direct averaging to the right-hand side of new system with respect to the fast phase $\delta$. Such averaging gives

$$
\begin{aligned}
\dot{K} & =0 \\
\dot{\theta} & =\varepsilon \Omega \sin \Delta \\
\dot{\Delta} & =-\varepsilon \Omega(\cos \Delta \tan \theta-\kappa \sin \theta) \\
\dot{\delta} & =\Omega+\frac{1}{2} \varepsilon \Omega\left[\cos \Delta \tan \left(\frac{\theta}{2}+\frac{\pi}{4}\right)+\kappa(1-\sin \theta)\right]
\end{aligned}
$$

where

$$
\kappa=\frac{3 \alpha K}{8 \Omega^{2}}
$$

The first equation in (9) shows that the energy parameter $K$ remains averagely constant regardless the magnitude of coupling and nonlinearity parameter $\varepsilon$. The fact that $K$ is constant justifies the use of quantities (7) and (8) for characterization of the energy exchange between the oscillators since neither the coupling nor nonlinear stiffness in (5) can accumulate the energy during one vibration cycle. In order to clarify physical meaning of the parameter $\kappa$, consider a single oscillator, $\ddot{u}+\Omega^{2} u+\alpha u^{3}=0$, whose mean (over the period) potential energy components, corresponding to linear and nonlinear stiffness terms, are $E_{\Omega}=\Omega^{2}<u^{2}>/ 2$ and $E_{\alpha}=\alpha<u^{4}>/ 4$, respectively. Assuming the harmonic temporal mode for the coordinate $u(t)$, and taking into account (8) and (10), gives

$$
\kappa=\frac{E_{\alpha}}{E_{\Omega}}
$$

Therefore, $\kappa$ characterizes the strength of nonlinearity in terms of its relative energy capacity during one vibration cycle. Taking into account (9) and (10), gives also $\dot{\kappa}=0$. Further complete description of the dynamics can be conducted now in terms of the two phase shift parameters, $\Delta(t)$ and $\theta(t)$, whereas the fast phase $\delta(t)$ is obtained by integration from the last equation in $(9)$. 


\section{The EP oscillator}

It can be shown by inspection that system (9) has the integral as follows

$$
-\cos \Delta \cos \theta+\frac{1}{2} \kappa \cos ^{2} \theta \equiv G=\text { const. }
$$

Taking into (12) and eliminating the phase $\Delta$ from the second and third equations of system (9), gives a single strongly nonlinear conservative oscillator with respect to the coordinate $\theta$ in the form $[7]$

$$
\ddot{\theta}+(\varepsilon \Omega)^{2}\left(G^{2} \frac{\tan \theta}{\cos ^{2} \theta}-\frac{1}{8} \kappa^{2} \sin 2 \theta\right)=0
$$

Note that the quantity $G$ in equation (13) remains constant only on a fixed dynamic trajectory in the plane $\theta-\Delta$ but may vary from one trajectory to another. Therefore the number $G(12)$ must be calculated first by fixing some point $\left\{\theta_{0}, \Delta_{0}\right\}$ on the trajectory. Then equation (13) can be solved by making sure that the initial condition $\{\theta(0), \dot{\theta}(0)\}$ corresponds to the fixed trajectory according to the second equation in (9). The parameter $\kappa$ however can be chosen independently on the dynamics in the plane $\theta-\Delta$. According to (7), equation (13) constitutes a principal equation describing the energy exchange between oscillators (5). Moreover, if the function $\theta(t)$ is known, then other two phase variables, $\Delta$ and $\delta$, are obtained from system (9) by differentiation and integration.

It is shown therefore that the entire system (9) is exactly solvable in quadratures since general solution of equation (13) can be obtained from its 'energy' integral, $H_{\theta}=$ const.,

$$
H_{\theta} \equiv \frac{1}{2} \dot{\theta}^{2}+(\varepsilon \Omega)^{2}\left(\frac{1}{2} G^{2} \tan ^{2} \theta+\frac{1}{16} \kappa^{2} \cos 2 \theta\right)
$$

The dynamics of oscillator (14) essentially depend on the shape of its potential energy within the interval $-\pi / 2<\theta<\pi / 2$. In particular, if the parameter $\kappa$ is small enough, then oscillator (13) has one stable (by Lyapunov) equilibrium position at $\theta=0$. However, high nonlinearity levels of system (1), associated with relatively large $\kappa$, can make the equilibrium position $\theta=0$ unstable by generating two stable equilibrium positions. Such kind of bifurcation provides sufficient conditions for energy localization effect as discussed in Section 5.

\section{Linear case}

When the original system is linear $(\alpha=0 \Longrightarrow \kappa=0)$, still strongly nonlinear equation (13) possesses explicit analytical solution within the class of elementary functions

$$
\theta(t)=\arcsin \left[\sin \theta_{0} \sin \phi(t)\right]
$$

where $\theta_{0}$ is the amplitude of $\theta$, whereas another constant can be introduced into the phase

$$
\phi(t)=\varepsilon \sec \theta_{0}|G| \Omega t
$$

as an arbitrary temporal shift admitted by equation (13). Such type of explicit solution has been known for quite a long time with no relation to any physical system [11], however it was used recently in some physical and mechanical applications in a phenomenological way [12], [13]. Although solution (15) holds only for the linearized model (5), it nevertheless helps to clarify specifics of the behavior of phase variables in nonlinear cases. In particular, substituting (15) in the second equation of $(9)$, gives

$$
\Delta(t)=\arcsin \left[\frac{|G| \tan \theta_{0} \cos \phi(t)}{\sqrt{1-\sin ^{2} \theta_{0} \sin ^{2} \phi(t)}}\right]
$$

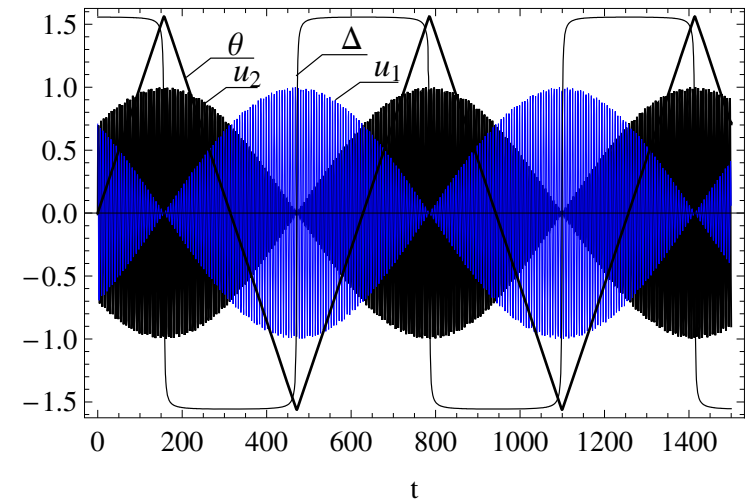

Fig. 1. Exact solutions for the beat dynamics of two identical linearly coupled harmonic oscillators and associated phase variables of the EP oscillator; a highly intensive energy exchage.

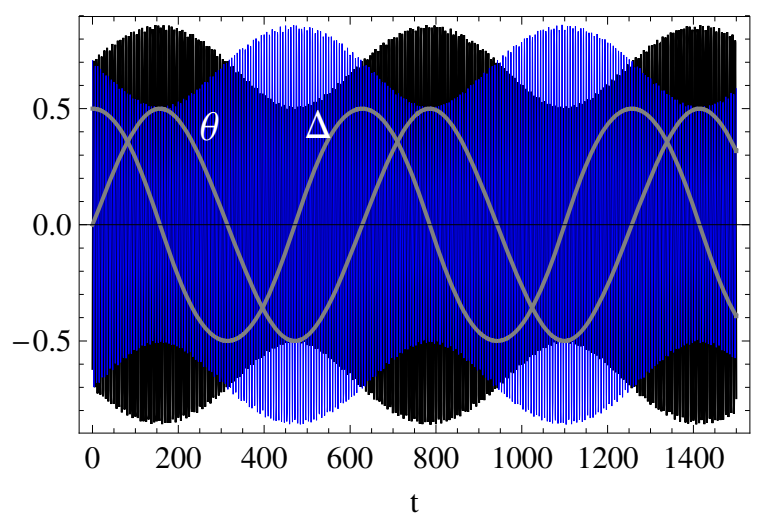

Fig. 2. Same as Fig.1; a moderate energy exchange.

Fig. 1 illustrates the relationship between the beat dynamics of linearized system (5) and the corresponding phase variables, $\theta(t)$ and $\Delta(t)$. The coordinates $u_{i}(t),(i=1,2)$ represent exact analytical solution under the initial conditions given by (6) at $t=0$. The integral $G$ is calculated at the amplitude point ${ }^{1}$,

1 The notation $\theta_{0}$ should not be confused with the initial value, which is $\theta(0)=0$, according to the present form of the solution. 
$\theta_{0}=\pi / 2-0.01$, which is slightly below its maximal possible magnitude $\pi / 2$. Since $\Delta=0$ as $\theta=\theta_{0}$, then $G=-\cos \theta_{0}$ and therefore $\phi(t)=\varepsilon \Omega t$. Other parameters are taken as $\varepsilon=0.01, \Omega=1.0, K=1.0$ and $\delta(0)=0$. As follows from Fig.1, the behavior of phase variables $\theta$ and $\Delta$ resembles smoothed time histories of the coordinate and velocity of a simple impact oscillator. As mentioned in Introduction, this fact was noticed first by Manevitch [5], [8] based on the analysis of phase equations similar to (9) however obtained in a different way after complexification of the coordinates. In particular, it was found that the impact limit' corresponds to the most intensive energy exchange between the oscillators when each of the oscillators periodically hosts the total energy of the system. It is seen now that, in the linearized case, such asymptotic follows directly from exact solutions, (15) and (16),

$$
\begin{gathered}
\theta(t) \rightarrow \arcsin (\sin \varepsilon \Omega t)=\frac{\pi}{2} \tau\left(\frac{2 \varepsilon \Omega t}{\pi}\right) \\
\Delta(t) \rightarrow \arcsin \left(\frac{\cos \varepsilon \Omega t}{|\cos \varepsilon \Omega t|}\right)=\frac{\pi}{2} e\left(\frac{2 \varepsilon \Omega t}{\pi}\right)
\end{gathered}
$$

as $\theta_{0} \rightarrow \pi / 2$

where $\tau(z)$ and $e(z)$ are triangular sine and rectangular cosine wave functions whose amplitude is unity and the period is normalized to four in order to provide the basic relationships of nonsmooth temporal transformations ${ }^{2}, \tau^{\prime}(z)=e(z)$ and $e^{2}(z)=1$ [7].

Now substituting (15) in (7), gives

$$
\begin{aligned}
& E_{1}=\frac{1}{2} E_{0}\left(1-\sin \theta_{0} \sin \varepsilon \Omega t\right) \\
& E_{2}=\frac{1}{2} E_{0}\left(1+\sin \theta_{0} \sin \varepsilon \Omega t\right)
\end{aligned}
$$

Taking into account (7) and (18), gives the corresponding energy partition index

$$
\begin{aligned}
P(t) & =\frac{E_{1}(t)-E_{2}(t)}{E_{1}(t)+E_{2}(t)} \\
& =-\sin \theta=-\sin \theta_{0} \sin \varepsilon \Omega t
\end{aligned}
$$

According to definition (19), the number $P=0$ indicates equipartition, $E_{1}=E_{2}$, whereas $P=1$ or $P=-1$ correspond to the case when all the energy belongs to either first or second oscillator, respectively. As follows from (19), such states can be reached only in the limit case (17), when $\theta_{0}=\pi / 2$.

In case of small amplitudes $\left|\theta_{0}\right| \ll \pi / 2$, corresponding to a moderate energy exchange, solutions (15) and (16) are approaching another simple limit of harmonic temporal shapes as illustrated by Fig. 2, where the amplitude is $\theta_{0}=0.5$. This also follows directly from the linearization of equation (13) near zero $\theta=0$.

2 The corresponding manipulations will be illustrated in the extended text.

\section{Nonlinear localization phenomenon}

In the nonlinear case, $\kappa \neq 0$, equation (13) still admits exact analytical solution in implicit form, which can be found from (14) by integration. However, assuming that $|\theta| \ll \pi / 2$, consider the following qubic approximation

$$
\ddot{\theta}+(\varepsilon \Omega)^{2}\left[\left(G^{2}-\frac{\kappa^{2}}{4}\right) \theta+\frac{1}{6}\left(8 G^{2}+\kappa^{2}\right) \theta^{3}\right]=0
$$

As follows from (7), the equilibrium point $\theta=0$ of oscillator (20) corresponds to equal energy distribution, under which the original model (1) remains in one of its two symmetric nonlinear normal modes. So when the linear stiffness is positive, equation (20) has periodic solutions describing the energy exchange between oscillators (5) near either in-phase or out-ofphase mode. However, the equal energy distribution, associated with the equilibrium $\theta=0$, becomes unstable if the linear stiffness is negative, $G^{2}-\kappa^{2} / 4<0$. In this case two new stable equilibria surrounded by separatrix loops occur near the unstable equilibrium. This indicates onset of nonlinear local modes of the original system (5) with a sustainable disbalance in the energy distribution despite of the perfect symmetry of system (1). In terms of the present notations, the condition of negative linear stiffness can be represented in the form [7]

$$
f_{1} \equiv-\frac{\kappa P_{0}^{2}}{2 \sqrt{1-P_{0}^{2}}}<\cos \Delta(0)<\frac{\kappa\left(2-P_{0}^{2}\right)}{2 \sqrt{1-P_{0}^{2}}} \equiv f_{2}
$$

where $P_{0}=P(0)$ is the initial energy partition index.

Condition (21) constitutes a necessary condition of localization because it does not guarantee that the dynamics will be trapped inside one of the separatrix loops. The corresponding sufficient condition is obtained from the energy integral of oscillator (20) in either of the following two inequalities, first of which never holds at positive $\kappa$,

$$
\begin{aligned}
& \cos \Delta(0)<-\frac{2+\kappa P_{0}^{2}}{2 \sqrt{1-P_{0}^{2}}} \equiv g_{1} \\
& \cos \Delta(0)>\frac{2-\kappa P_{0}^{2}}{2 \sqrt{1-P_{0}^{2}}} \equiv g_{2}
\end{aligned}
$$

Both estimates (21) and (22) are also valid locally, in the neighborhood of zero $\theta=0$, for strongly nonlinear oscillator (14).

The above conditions (21) and (22) must be considered under the obvious constraint $|\cos \Delta(0)| \leq 1$. Fig. 3 illustrates a relatively low nonlinearity case, when localization is impossible. The solid lines represent the boundary functions, introduced in (21) and (22), whereas the couple of dashed lines indicates the rectangular area within which solutions of inequalities (21) and (22) the above mentioned constraint. When the strength of nonlinearity $\kappa$ is increased, the line $f_{2}$ moves upward, whereas the line $g_{2}$ moves downward. When passing one through another at about $\kappa=1$, two small areas of localization occur as shown in Fig. 4. Note that, in both localization areas, the initial 


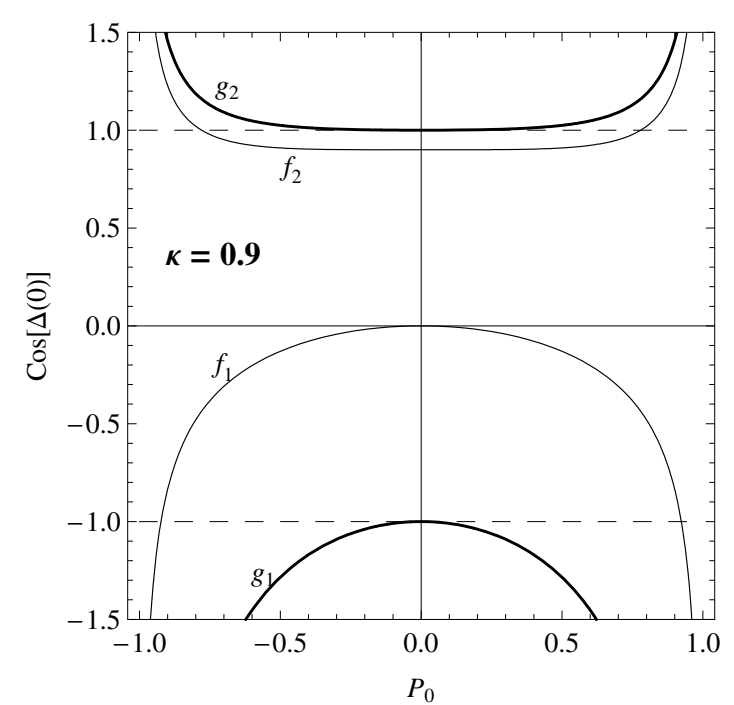

Fig. 3. The plane of initial phase versus energy partition showing no localization area at relatively low nonlinearity $\kappa$; dashed horizontal lines bound the allowed region $|\cos [\Delta(0)]| \leq 1$.

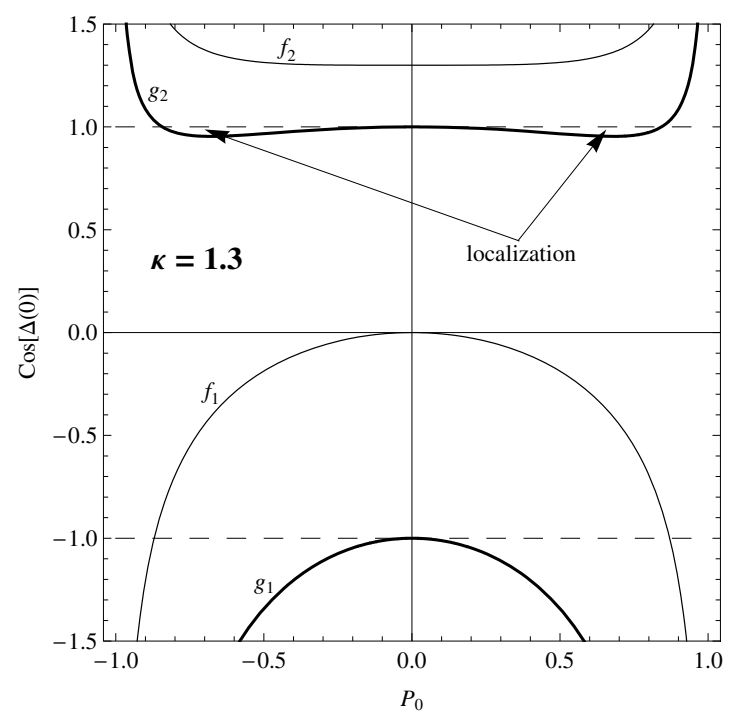

Fig. 4. The plane of initial phase versus energy partition showing two localization areas on higher nonlinearity level $\kappa$.

phase angle $\Delta$ lays in the neighborhood of zero. Therefore, according to (6), the localized modes branch out of the out-of-phase modes as the nonlinearity becomes sufficiently strong.

\section{Concluding remarks}

In this work, it is shown that the phase angle of energy distribution between two coupled Duffing oscillators, $\theta(t)$, is described by exactly solvable strongly nonlinear conservative (EP) oscillator. Moreover, such oscillator admits physically clear simplifications with approximate explicit solutions in terms of elementary functions. Note that the corresponding differential equation can be obtained also for the partition index $P(t)$ by using expression (19) as a substitution. It is essential that the parameters of EP oscillator are expressed through the integrals of system (9). As a result, initial conditions for the EP oscillator must comply with those of the original system through the phase coordinate transformation (6). Practically, by taking into account the second equation in (9) and expression (19), the initial conditions can be expressed first through the initial energy partition index $P$ and the phase $\Delta$ angle as $\theta(0)=-\arcsin P(0)$ and $\dot{\theta}(0)=\varepsilon \Omega \sin \Delta(0)$. Therefore, the direction of energy flow in the original system is completely predicted by the initial energy distribution and the initial phase shift between the interacting oscillators. Obviously adding a weak dissipation to the system cannot affect the above conclusion in a short term. However, the long-term dynamics may experience some qualitative changes as the strength of nonlinearity $\kappa$ slowly decays due to the total system energy dissipation.

\section{References}

1. A. F. Vakakis, L. I. Manevitch, Y. V. Mikhlin, V. N. Pilipchuk, and A. A. Zevin, Normal modes and localization in nonlinear systems (John Wiley \& Sons, New York 1996)

2. A. Kosevich and A. Kovalev, Introduction to Nonlinear Physical Mechanics (Naukova Dumka, Kiev 1989), in Russian

3. D. D. Holm and P. Lynch, SIAM J. Applied Dynamical Systems 1, (2002) 44-64.

4. L.I. Manevitch, Proc. 8th Conference on Dynamical Systems - Theory and Applications, Lodz, 2005 1, (2005) 289.

5. L.I. Manevitch, Arch. Appl. Mech. 77, (2007) 301312

6. V.N. Pilipchuk, Dokl. Akad. Nauk Ukrain. SSR Ser. A, No.4, (1988), 37-40.

7. V.N. Pilipchuk, Nonlinear Dynamics: between linear and impact limits (Springer-Verlag, Berlin Heidelberg 2010)

8. L. I. Manevitch and A. Musienko, 2nd International Conference on Nonlinear Normal Modes and Localization in Vibrating Systems, Samos, Greece, June 19-23, (2006) 25-26

9. L.I. Manevitch and V.V. Smirnov, arXiv, 0903.5455v1, (2009)

10. Y. Starosvetsky and L. I. Manevitch, Physical Review E 83,(2011) 046211

11. H. Kauderer, Nichtlineare Mechanik (Springer, Berlin 1958)

12. S. V. Nesterov, Proceedings of Moscow Institute of Power Engineering 357,(1978) 68-70

13. M. F. Dimentberg and A. S. Bratus, R. Soc. Lond. Proc. Ser. A Math. Phys. Eng. Sci. 456,(2002) 2351-2363, 2000. 